Article

\title{
Associations between Fatty Acid Intake and Status, Desaturase Activities, and FADS Gene Polymorphism in Centrally Obese Postmenopausal Polish Women
}

\author{
Agata Muzsik ${ }^{1}$, Joanna Bajerska ${ }^{1}$, Henryk H. Jeleń ${ }^{2}$, Anna Gaca ${ }^{2}$ and Agata Chmurzynska ${ }^{1, *(\mathbb{D})}$ \\ 1 Institute of Human Nutrition and Dietetics, Poznan University of Life Sciences, 60-624 Poznan, Poland; \\ agata.muzsik@up.poznan.pl (A.M.); joanna.bajerska@up.poznan.pl (J.B.) \\ 2 Institute of Food Technology of Plant Origin, Poznan University of Life Sciences, 60-637 Poznan, Poland; \\ henryk.jelen@up.poznan.pl (H.H.J.); anna.gaca@up.poznan.pl (A.G.) \\ * Correspondence: agata.chmurzynska@up.poznan.pl; Tel.: +48-61-846-6181
}

Received: 12 July 2018; Accepted: 8 August 2018; Published: 10 August 2018

\begin{abstract}
Fatty acid (FA) status is associated with the risk of several diet-related diseases. Since postmenopausal women are at increased risk of cardiometabolic disturbances, determinants of FA metabolism should be fully understood in this group. We hypothesize that FA metabolism in postmenopausal Polish women may depend on current macronutrient intake and on fatty acid desaturase (FADS) gene polymorphism. One-hundred-and-twenty-eight postmenopausal women with central obesity were recruited to the study and their dietary intake, FA composition in red blood cells (RBC), and rs174556, rs174561, rs174547, and rs3834458 polymorphism of the FADS gene were analyzed. Higher levels of 18:2n-6t level in RBC were associated with higher protein or fat intake or with lower carbohydrate intake. The minor allele carriers of rs174561 of the fatty acid desaturase 1 (FADS1) gene had 9.7\% lower concentration of 20:4n-6 in RBC $(p<0.05)$, but there were no other associations between other FA in RBC levels and FADS1 or fatty acid desaturase 2 (FADS2) polymorphisms. The mean D5D value was $15.3-17.9 \%$ lower in the minor allele carriers of each SNPs. We concluded that protein and carbohydrate intake may be associated with FA concentrations in RBC in centrally obese postmenopausal Polish women. The D5D value may be affected by FADS1 or FADS2 polymorphism.
\end{abstract}

Keywords: fatty acids; dietary intake; fatty acid desaturase (FADS) gene; postmenopausal women

\section{Introduction}

Fat and fatty acid (FA) composition in diet has a significant effect on health, and thus plays an important role in the development of noncommunicable diseases, such as overweight, obesity, metabolic syndrome (MetS), and nonalcoholic fatty liver disease. Postmenopausal women, who show slower energy metabolism, are more prone to these metabolic disorders, especially disturbances of lipid metabolism [1-5]. Additionally, the postmenopausal diet is characterized by a low intake of vegetables and fiber and a high intake of protein and animal fat, which results in higher intakes of saturated fatty acids (SFA) and lower unsaturated fatty acids (unSFA) [6-9]. Similarly, in postmenopausal Polish women with dyslipidemia, lower polyunsaturated fatty acids (PUFA) intake and higher SFA intake have also been observed [10].

FA nutritional status and the metabolic health of postmenopausal women depend not only on the dietary intake of FAs, but also on their endogenous synthesis (Figure 1). The metabolism of long-chain polyunsaturated fatty acids (LC-PUFAs) consists of alternate reactions of desaturation and elongation. It also depends on dietary precursors, which mammals cannot produce. $\alpha$-linolenic 
acid (ALA, 18:3n-3) is a precursor in the synthesis of eicosapentaenoic acid (EPA, 20:5n-3) and docosahexaenoic acid (DHA, 22:6n-3), while linoleic acid (LA, 18:2n-6) is a precursor in the synthesis of arachidonic acid (AA, 20:4n-6). LC-PUFA pathways are limited by the enzymes 5- $\Delta$-desaturase (D5D) and 6- $\triangle$-desaturase (D6D), which are encoded by fatty acid desaturase $1(F A D S 1)$ and 2 (FADS2) genes, respectively (Figure 1) [11-13].

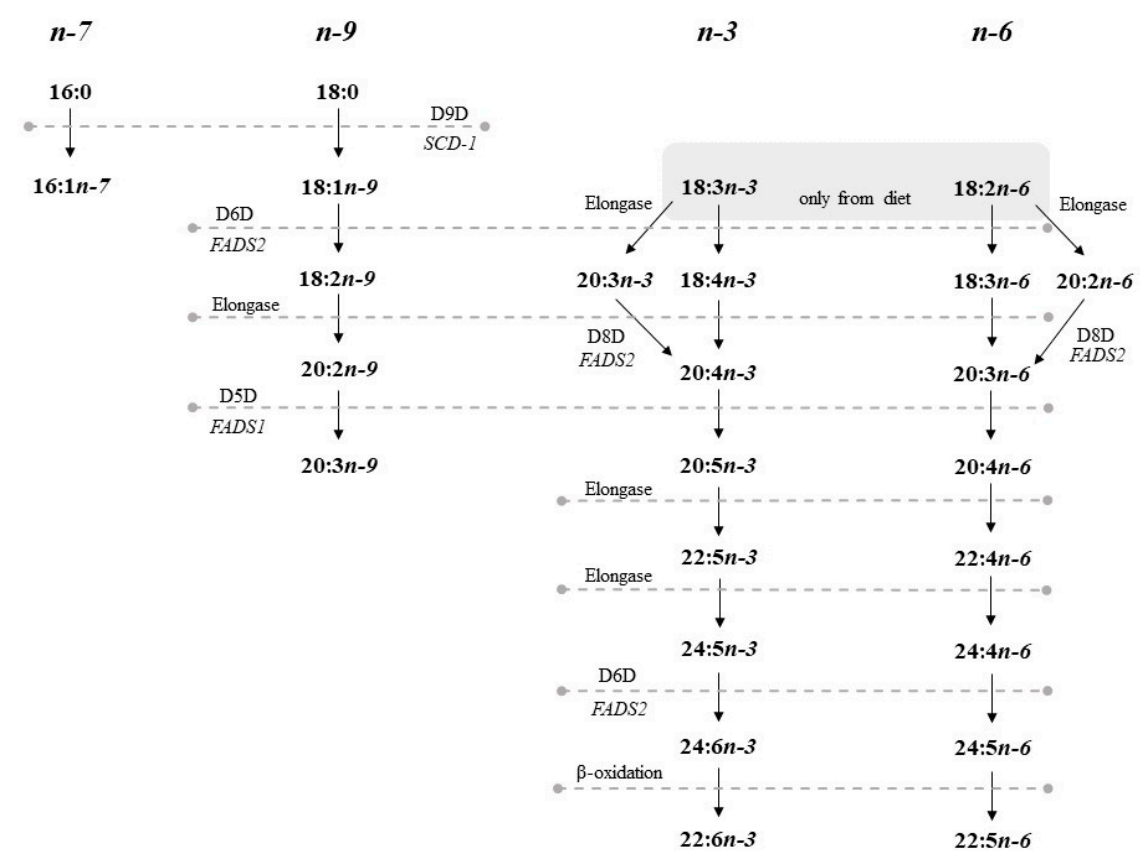

Figure 1. Endogenous pathways of polyunsaturated fatty acids (PUFA) metabolism in mammals. D5D: Delta-5 desaturase; D6D: Delta-6 desaturase; D8D: Delta-8 desaturase; D9D: Delta-9 desaturase; SCD-1: Stearyl-CoA desaturase 1; FADS1: Fatty acid desaturase 1; FADS2: Fatty acid desaturase 2; PUFA: Polyunsaturated fatty acids.

The activities of D5D and D6D depend on different factors, including genetic polymorphism, aging, dietary PUFA, metabolic disorders, and blood lipids [12,14-19]. Enzyme activity can also be affected by single-nucleotide polymorphisms (SNPs) of FADS1 and FADS2. FADS genotypes account for up to $28 \%$ of the variability in FA concentrations in serum phospholipids [11]. There have been a number of studies showing associations between variations in FADS (rs174556, rs174547, rs174561, and rs3834458) and FA status, and the majority reported that minor allele carriers have decreased synthesis of LC-PUFA with higher proportions of ALA to EPA and DHA and LA to AA [11-13,20-26].

There have been several studies to analyze FA metabolism, FA enzyme activity, or FADS gene-cluster polymorphism in adults [11-13], but these have focused on patients with MetS or diabetes [27], pregnant or lactating women [28], elderly people [29], or subjects with cardiovascular disease [30]. There have only been a few studies to consider all these factors together [11-14,20,30-32]; these were cross-sectional studies that examined both women and men together, without age subgroups. For this reason, there is still a lack of data on FA status in postmenopausal women. Since FA status is associated with the risk of several diet-related noncommunicable diseases, the determinants of FAs metabolism and their concentrations in blood and tissues should be fully understood in different populations. This is of special importance in postmenopausal women, where lipid metabolism abnormalities are often observed.

We hypothesize that FA metabolism in postmenopausal Polish women depends on current protein, carbohydrate, fat, and FA intake, and on FADS gene polymorphism. The primary aim of this study is to investigate the relationship between FA intake or FADS1 and FADS2 polymorphism with the LC-PUFA metabolism. A secondary aim is to determine the effect of FADS gene polymorphism on 
the activities of fatty acid enzymes, such as D5D and D6D. To this end, FA intake, FA profile in red blood cells (RBC), and FADS1 and FADS2 genotype were examined in a group of postmenopausal Polish women.

\section{Materials and Methods}

\subsection{Subjects}

The subjects were recruited in 2014 through advertisements in local newspapers [33]. Women in postmenopausal period and under 70 years of age were included for the study. The postmenopausal period was defined as at least 1 year since the last menstrual period plus a follicle-stimulating hormone concentration of $30 \mathrm{IU} / \mathrm{L}$ at screening. The exclusion criteria were participation in a weight loss therapy or weight fluctuation in the 6 months prior to the current study, intolerance or food allergies, a history of heart disease, insulin-dependent diabetes or type 2 diabetes, kidney disease, hypothyreosis, chronic inflammatory disease, liver disease, osteoporosis, any type of cancer, and tobacco smoking. Additionally, women were excluded if they were undergoing hormone replacement therapy or medication for a psychiatric disorder. Poznan Medical University approved the protocol for the study (number 603/14). All participants gave their written informed consent before enrollment to the study.

\subsection{Dietary Assessment}

Dietary intake was assessed using a 3-day food diary in which the participants were clearly instructed to record information on nonconsecutive days ( 2 weekdays and 1 weekend day) regarding their food, beverage and supplements intake [34], using household measures. The food and beverage quantities thus obtained were converted into grams and milliliters and computed using the dietary analysis software Dieta 5.0 (National Food and Nutrition Institute, Warsaw, Poland); the average intake of nutrients (protein, fat, carbohydrates, FA, cholesterol) and energy was thus calculated. The Polish dietary standards were used to assess adequate intakes [35].

\subsection{Anthropometry}

The anthropometric data included body weight, height, body mass index $\left(\mathrm{BMI}=\right.$ weight $/$ height $\left.^{2}\right)$, and waist circumference. Height was measured to the nearest $0.5 \mathrm{~cm}$ by stadiometer WPT $100 / 200$ OW (RadWag, Poznan, Poland). Body weight was measured to the nearest $0.1 \mathrm{~kg}$ with subjects in a bathing suit following an overnight fast, using a calibrated scale included in the Bod Pod (Cosmed, Rome, Italy). Waist circumference was measured at the midpoint between the lowest rib and the top of the iliac crest using nonelastic tape. This measurement was performed by a single evaluator. Women with waist circumference $\geq 80 \mathrm{~cm}$ were considered centrally obese [36].

\subsection{Physical Activity}

Physical activity level was assessed using the short version of the International Physical Activity Questionnaire (IPAQ), which has been validated for youths and adults. Moderate and intensive physical activity, work, active commuting, and household activity were calculated from their durations in the previous week [37].

\subsection{Analysis of Fatty Acid Profile in Erythrocytes}

Erythrocytes were separated from whole blood by density gradient centrifugation by centrifuge 5702R (Eppendorf, Hamburg, Germany), washed with phosphate buffer saline (PBS), and stored at $-80{ }^{\circ} \mathrm{C}$. FAs were extracted using the modified Folch method [38] —briefly, the Folch reagent (2:1 chloroform: methanol) for extraction of lipids from cells, butylated hydroxytoluene as an antioxidant and internal standard: deuterated myristic $-\mathrm{d}_{27}$ acid $\left(\mathrm{d}_{27} \mathrm{C} 14: 0\right)$ in chloroform were added to weighted samples and the mixture was centrifuged. The approximate $2 \mathrm{~mL}$ of the lipid-containing 
chloroform phase was removed and derivatized with $0.5 \mathrm{~mL}$ of $0.5 \mathrm{M}$ potassium hydroxide in methanol and $1 \mathrm{~mL}$ of boron trifluoride methylation by heating at $70^{\circ} \mathrm{C}$. Fatty acid methyl esters (FAME) were extracted into $4 \mathrm{~mL}$ of hexane and washed out with $2 \mathrm{~mL}$ of distilled water. The organic (hexane) phase was then transferred into $2 \mathrm{~mL}$ vials and subsequently analyzed by gas chromatography. The analysis was performed on a Hewlett-Packard 6890 gas chromatograph (Wilmington, DE, USA) equipped with a split-splitless injector and a flame ionization (FID) detector. FAME were separated using a SelectFame column $(50 \mathrm{~m} \times 0.25 \mathrm{~mm} \times 0.25 \mu \mathrm{m}$, Agilent Technologies, Santa Clara, CA, USA identified by comparison with available FAME standards (Supelco, Bellefonte, PA, USA). Fatty acid content was calculated by comparing individual peak areas with the peak area of an internal standard and recalculated based on sample weight. Response factors (FID) for particular fatty acids versus internal standards were assumed to be unity. The concentration of fatty acids in RBC was expressed as $\mu \mathrm{g} / \mathrm{mL}$.

\subsection{Desaturase and Elongase Activities}

Desaturase and elongase activities were calculated using product-to-precursors ratios. The D5D and D6D were calculated using 20:4n-6/20:3n-6 and 18:3n-6/18:2n-6 ratios, respectively. The combined effects of desaturase and elongase activities ratios were calculated as follows: $20: 4 n-6 / 18: 2 n-6$ and 22:4n-6/18:2n-6 in n-6 PUFA metabolism and 20:5n-3/18:3n-3, 22:6n-3/18:3n-3 and 22:6n-3/20:5n-3 in n-3 PUFA metabolism.

\subsection{Genotyping}

The rs174556, rs174547, and rs174561 polymorphisms of the FADS1 gene and the rs3834458 polymorphism of the FADS2 gene were selected for analysis. The blood was collected into tubes containing EDTA, and the DNA isolated from fresh blood using a NucleoSpin Blood kit (Macherey-Nagel, Düren, Germany). Genotyping was performed with the use of hybridizing probes designed and synthesized by Tib MolBiol (Tib Molbiol, Berlin, Germany), as shown in Table 1, on a LightCycler 480 instrument (Roche, Basel, Switzerland). One probe was labeled with fluorescein and the other with LC640 dye. PCR was run using $10 \mu \mathrm{L}$ reaction mix at a primer concentration of $0.5 \mu \mathrm{M} / \mu \mathrm{L}$ and a probe concentration of $0.15 \mu \mathrm{M} / \mu \mathrm{L}$. The reaction was performed in 96 -well format in a total reaction volume of $10 \mu \mathrm{L}$, using $20 \mathrm{ng}$ of genomic DNA. The cycling profile was $95^{\circ} \mathrm{C}$ for $10 \mathrm{~min}$, followed by 45 cycles at $95{ }^{\circ} \mathrm{C}$ for $10 \mathrm{~s}, 61^{\circ} \mathrm{C}$ for $10 \mathrm{~s}$, and $72{ }^{\circ} \mathrm{C}$ for $15 \mathrm{~s}$. Genotypes were analyzed using a melting curve method.

Table 1. Primer and probe sequences used in the FADS genotyping assay.

\begin{tabular}{cccc}
\hline Gene & SNP & Primers & Probes \\
\hline \multirow{2}{*}{ FADS1 } & rs174556 & 5'ACAAGGGCCTTGTGAAGAAGT & \multirow{2}{*}{ 5'GAGTCTAGATGGaATCACAGTCATAGT-FL } \\
& $(\mathrm{C}>\mathrm{T})$ & 5'GCCTGTGACCTCATGACTATGC & \\
& rs174561 & 5'GCACCACACATACGGACCAAT & \multirow{2}{*}{ 5' GCATCCCCGGCCCCA-FL } \\
& $(\mathrm{T}>\mathrm{C})$ & 5'GGGTCAACCAGAGTGACCACTC & \\
& rs174547 & 5'TGGGTGACACAGATGAACCATATTC & \multirow{2}{*}{ 5'CTACGCACCCTTTTCAATAGTTG-FL } \\
& $(\mathrm{T}>\mathrm{C})$ & 5'GGCTAATGAGAAAATGCTGTTGG & \\
\hline \multirow{2}{*}{ FADS2 } & rs3834458 & 5'TTACTGAGACCAGGGCAAGGAC & \multirow{2}{*}{ 5'TCAGACAATCTT_GAAAAGAATTGC } \\
& $($ delT $)$ & 5'CGGCAGTCGAGACTCCAGTATC & \\
\hline
\end{tabular}

FADS: Fatty acid desaturase; SNP: Single-nucleotide polymorphism; FADS1: Fatty acid desaturase 1; FADS2: Fatty acid desaturase 2 .

\subsection{Statistical Analysis}

Student's $t$-test was applied to compare the crude means. Adjustment of continuous variables was performed by linear regression and the models were adjusted for BMI, physical activity, and whether hypolipidemic or hypoglycemic medications were being taken. The models for desaturase activities were also adjusted for age and intake of enzyme precursors. The group was stratified by nutrient intake with low or high intake here meaning below or above the median values. Intake was 
considered as \% energy from a particular nutrient. Additionally, protein intake was considered as a categorical variable (inadequate or inadequate). Adequate protein intake means $\geq 1.2 \mathrm{~g}$ of protein $/ \mathrm{kg}$ of body mass/day. $p<0.05$ was considered statistically significant. Data were analyzed using Statistica software (StatSoft, Tulsa, OK, USA).

\section{Results}

\subsection{Subjects Characteristics}

One-hundred-and-forty-three women provided dietary data and 128 out of them provided blood samples. The mean age was $60.7 \pm 5.1$ years and the average BMI was $33.7 \pm 4.9 \mathrm{~kg} / \mathrm{m}^{2}$. All women had central obesity and the mean waist circumference was $105.2 \pm 9.6 \mathrm{~cm}$.

\subsection{Dietary Intake of Macronutrients and FA}

The characteristics of the macronutrient and FA intakes are described in Table 2. The mean total energy intake was $1699 \pm 59.5 \mathrm{kcal}$, which did not include energy from dietary fiber. The mean percent energy intakes from protein, fat, and carbohydrates were $17.1 \pm 0.4 \%, 28.2 \pm 0.7 \%$, and $53.9 \pm 0.9 \%$, respectively. The mean percent energy intake from SFA was 10.9\%, while those from monounsaturated fatty acids (MUFA) and PUFA were $11.8 \%$ and $4.1 \%$, respectively. The mean PUFA+MUFA/SFA ratio was $1.5 \pm 0.04$, and more than $93.0 \%$ of participants had an excess intake of the percent energy from SFA.

Table 2. Dietary macronutrient and fatty acid intakes among postmenopausal women.

\begin{tabular}{|c|c|c|c|c|c|c|}
\hline \multirow{2}{*}{ Macronutrient. } & \multicolumn{6}{|c|}{ Daily Intake } \\
\hline & Mean \pm SEM & Median & Quartile 1 & Quartile 2 & Quartile 3 & Quartile 4 \\
\hline Total protein (g) & $67.6 \pm 1.6$ & 66.5 & $45.5 \pm 1.2$ & $61.2 \pm 0.6$ & $71.0 \pm 0.5$ & $93.3 \pm 2.6$ \\
\hline Total carbohydrates $(\mathrm{g})^{\mathrm{a}}$ & $255.7 \pm 11.1$ & 229.5 & $128.6 \pm 3.8$ & $191.6 \pm 3.3$ & $265.6 \pm 3.9$ & $437.2 \pm 20.5$ \\
\hline Total lipids $(\mathrm{g})$ & $52.4 \pm 2.0$ & 47.4 & $27.4 \pm 0.8$ & $41.0 \pm 0.7$ & $57.4 \pm 0.9$ & $83.8 \pm 3.6$ \\
\hline Total SFAs (g) & $20.1 \pm 0.9$ & 18.4 & $9.9 \pm 0.3$ & $15.2 \pm 0.3$ & $21.7 \pm 0.3$ & $33.7 \pm 1.8$ \\
\hline $4: 0(\mathrm{mg})$ & $315.4 \pm 26.7$ & 258.3 & $90.7 \pm 6.3$ & $201.1 \pm 4.8$ & $315.8 \pm 5.6$ & $654.2 \pm 79.3$ \\
\hline $6: 0(\mathrm{mg})$ & $211.6 \pm 16.8$ & 171.6 & $71.7 \pm 4.4$ & $135.0 \pm 2.9$ & $210.8 \pm 3.7$ & $428.7 \pm 49.6$ \\
\hline $8: 0(\mathrm{mg})$ & $153.2 \pm 10.7$ & 129.9 & $56.7 \pm 3.0$ & $104.5 \pm 2.4$ & $156.4 \pm 2.7$ & $295.3 \pm 30.2$ \\
\hline 10:0 (mg) & $378.6 \pm 26.1$ & 318.6 & $136.0 \pm 6.4$ & $258.0 \pm 5.9$ & $379.9 \pm 6.6$ & $740.5 \pm 70.7$ \\
\hline $12: 0(\mathrm{mg})$ & $558.3 \pm 35.6$ & 468.3 & $209.5 \pm 10.6$ & $379.0 \pm 8.1$ & $578.4 \pm 8.8$ & $1067.0 \pm 91.6$ \\
\hline $14: 0(\mathrm{~g})$ & $2.2 \pm 0.1$ & 1.9 & $0.9 \pm 0.03$ & $1.6 \pm 0.03$ & $2.2 \pm 0.04$ & $3.9 \pm 0.3$ \\
\hline 15:0 (mg) & $252.4 \pm 14.4$ & 215.9 & $95.1 \pm 5.0$ & $178.6 \pm 3.9$ & $264.4 \pm 5.0$ & $471.9 \pm 32.0$ \\
\hline $16: 0(\mathrm{~g})$ & $11.2 \pm 0.5$ & 10.3 & $5.7 \pm 0.2$ & $8.7 \pm 0.2$ & $12.1 \pm 0.2$ & $18.3 \pm 0.8$ \\
\hline 17:0 (mg) & $173.8 \pm 10.6$ & 151.5 & $66.7 \pm 3.4$ & $121.0 \pm 3.0$ & $179.5 \pm 2.9$ & $328.0 \pm 26.4$ \\
\hline $18: 0(\mathrm{~g})$ & $4.6 \pm 0.2$ & 4.1 & $2.0 \pm 0.1$ & $3.3 \pm 0.1$ & $5.0 \pm 0.1$ & $8.1 \pm 0.4$ \\
\hline 20:0 (mg) & $75.7 \pm 4.8$ & 62.9 & $20.0 \pm 1.2$ & $47.9 \pm 1.2$ & $81.3 \pm 2.0$ & $153.9 \pm 9.0$ \\
\hline Total MUFA (g) & $20.4 \pm 08$ & 19.2 & $9.7 \pm 0.3$ & $15.8 \pm 0.4$ & $22.3 \pm 0.4$ & $33.7 \pm 1.3$ \\
\hline $14: 1(\mathrm{mg})$ & $193.0 \pm 13.3$ & 164.7 & $74.0 \pm 3.8$ & $132.7 \pm 2.8$ & $196.5 \pm 2.9$ & $370.4 \pm 37.4$ \\
\hline $15: 1(\mathrm{mg})$ & $67.1 \pm 5.9$ & 48.3 & $21.8 \pm 1.4$ & $40.6 \pm 0.9$ & $65.4 \pm 1.6$ & $140.8 \pm 18.0$ \\
\hline $16: 1(\mathrm{~g})$ & $1.2 \pm 0.1$ & 1.1 & $0.5 \pm 0.03$ & $1.0 \pm 0.02$ & $1.4 \pm 0.02$ & $2.1 \pm 0.1$ \\
\hline 17:1 (mg) & $111.6 \pm 9.2$ & 90.1 & $36.6 \pm 2.1$ & $69.7 \pm 1.8$ & $111.1 \pm 1.9$ & $228.7 \pm 27.3$ \\
\hline $18: 1(\mathrm{~g})$ & $18.3 \pm 0.8$ & 16.8 & $8.7 \pm 0.3$ & $14.1 \pm 0.3$ & $19.9 \pm 0.3$ & $30.6 \pm 1.1$ \\
\hline $20: 1(\mathrm{mg})$ & $239.1 \pm 15.9$ & 191.3 & $73.6 \pm 4.8$ & $155.0 \pm 3.0$ & $239.5 \pm 6.2$ & $488.5 \pm 35.2$ \\
\hline 22:1 (mg) & $156.2 \pm 21.3$ & 51.8 & $1.1 \pm 0.3$ & $23.7 \pm 2.3$ & $115.6 \pm 8.1$ & $483.2 \pm 54.2$ \\
\hline PUFA total (g) & $7.4 \pm 0.4$ & 6.1 & $3.8 \pm 0.1$ & $5.5 \pm 0.1$ & $7.3 \pm 0.1$ & $13.0 \pm 1.1$ \\
\hline $18: 2(\mathrm{~g})$ & $5.8 \pm 0.3$ & 4.9 & $3.0 \pm 0.1$ & $4.5 \pm 0.1$ & $5.8 \pm 0.1$ & $9.8 \pm 0.5$ \\
\hline $18: 3(\mathrm{~g})$ & $1.3 \pm 0.2$ & 0.8 & $0.5 \pm 0.01$ & $0.7 \pm 0.01$ & $1.0 \pm 0.02$ & $3.1 \pm 0.7$ \\
\hline 18:4 (mg) & $9.7 \pm 2.2$ & 0.0 & $0.0 \pm 0.0$ & $0.0 \pm 0.0$ & $2.8 \pm 0.3$ & $37.5 \pm 6.9$ \\
\hline $20: 3(\mathrm{mg})$ & $0.6 \pm 0.3$ & 0.0 & $0.0 \pm 0.0$ & $0.0 \pm 0.0$ & $0.0 \pm 0.0$ & $9.4 \pm 4.4$ \\
\hline $20: 4(\mathrm{mg})$ & $119.5 \pm 8.7$ & 91.0 & $30.7 \pm 2.3$ & $68.9 \pm 2.0$ & $123.1 \pm 4.1$ & $255.2 \pm 19.1$ \\
\hline $20: 5(\mathrm{mg})$ & $59.3 \pm 10.4$ & 11.3 & $0.2 \pm 0.1$ & $5.6 \pm 0.4$ & $27.0 \pm 2.1$ & $203.7 \pm 30.3$ \\
\hline $22: 5(\mathrm{mg})$ & $23.8 \pm 3.9$ & 5.9 & $0.1 \pm 0.1$ & $3.7 \pm 0.2$ & $11.4 \pm 0.5$ & $79.8 \pm 10.8$ \\
\hline $22: 6(\mathrm{mg})$ & $139.4 \pm 21.6$ & 50.3 & $9.6 \pm 1.0$ & $34.6 \pm 1.4$ & $82.4 \pm 3.5$ & $429.4 \pm 64.8$ \\
\hline
\end{tabular}

${ }^{a}$ Calculations of total carbohydrate intake did not include dietary fiber and resistant starch. SFA: Saturated fatty acids; MUFA: Monounsaturated fatty acids; PUFA: Polyunsaturated fatty acids; SEM: standard error of mean; $N=143$ 


\subsection{Associations between Macronutrient Intake and FA Composition In Erythrocyte Membranes}

We examined how macronutrient intake affected FA composition in erythrocyte membranes. Higher protein intake was associated with higher concentrations in RBC of $16: 1 n-7,18: 1 n-7 c, 18-1 n-5 c$, and 18:2n-6t ( $p=0.035,0.022,0.007$, and 0.001, respectively; see Table 3$)$. The opposite effect was observed for 17:1, 18:1n-7t, 22:1n-9, 22:4n-6 and 22:6n-3 in RBC. A lower intake of carbohydrates (below the median value) was significantly associated with the $21.5 \%$ higher level of $18: 2 \mathrm{n}-6 \mathrm{t}(p<0.05)$ and the 19.9\% lower level of 17:1 ( $p<0.05$; Table 3). Higher percentage energy intake from fat (above the median value) was associated with $26.0 \%$ higher concentration of $18: 2 \mathrm{n}-6 \mathrm{t}(p<0.05)$ and $9.0 \%$ lower concentration of 22:4n-6 ( $p<0.05$; Table 3). Moreover, no associations were seen between fat intake and other FA concentrations in erythrocyte membranes. We also examined the associations between percentage energy from SFA, PUFA, MUFA, and FA concentrations in RBC, and no general associations were found (Supplementary Materials, Tables S1-S3). The only significant result was an association between higher percentage energy from MUFA and a 10.8\% lower level of 22:5n-6 in RBC $(p<0.05$; Supplementary Materials, Table S2).

\subsection{Associations between FADS Genotype and FA Concentrations in Erythrocyte Membranes and Enzyme Activities}

The associations between FADS1 and FADS2 polymorphisms and FA in RBC are shown in Table 4. The rs174561 polymorphism of FADS1 was associated with 20:4n-6 concentration in $\operatorname{RBC}(p<0.05)$, but there were no other associations between other FAs in RBC levels and the FADS1 or FADS2 polymorphisms. Enzyme activities are described in Table 5. We also examined the associations between gene polymorphism and desaturase or elongase activities (Table 6). The D5D value was significantly lower (15.3-17.9\%) in subjects with at least one minor allele of any of the examined polymorphisms $(p<0.001)$. In addition, rs174561 and rs3834458 were associated with 22:6n-3/20:5n-3 ratio $(p<0.05)$, and women with at least one minor allele had over $12.0 \%$ higher values of this parameter (Table 6). However, the other indices (D6D, 20:4n-6/18:2n-6, 22:4n-6/18:2n-6, 20:5n-3/18:3n-3 and 22:6n-3/18:3n-3) were similar between genotype groups.

We also tested whether and how the dietary intake of protein (as well as enzyme substrates and products) affected enzyme activities. Protein intake affected desaturase activities and the 22:6n-3/20:5n-3 and the 22:6n-3/22:5n-3 ratio was lower in women who met their needs for protein. Specifically, 22:6n-3/20:5n-3: $4.49 \pm 0.17$ versus $4.01 \pm 0.16(p<0.05)$ and 22:6n-3/22:5n-3: $1.03 \pm$ 0.07 as against $0.78 \pm 0.06(p<0.05)$ in women who met and failed to meet the needs for protein, respectively. The same type of association was found for protein intake and lipogenic index (the ratio of 16:0 to $18: 2 n-6$ which reflects de novo lipogenesis $[39,40])(1.80 \pm 0.07$ vs. $0.78 \pm 0.06, p<0.05)($ data not shown). Moreover, there was no effect of the intake of a product or a substrate of the desaturases on those parameters. Additionally, associations between the examined polymorphisms and the desaturase activities were tested in the regression models. Each of the analyzed polymorphisms were associated with the D5D value, and possessing at least one minor allele was associated with about $25 \%$ higher D5D activity (Table 6). Moreover, rs174561 and rs3834458 were associated with the 22:6n-3/20:5n-3 ratio, and women with at least one minor allele had over $15 \%$ higher values of this parameter (Table 6). 
Table 3. Concentrations of erythrocyte fatty acids stratified by macronutrient intake.

\begin{tabular}{|c|c|c|c|c|c|c|c|c|c|}
\hline $\begin{array}{l}\text { Concentrations of FA } \\
\text { in } R B C(\mu \mathrm{g} / \mathrm{mL})\end{array}$ & Low Protein ${ }^{a}$ & High Protein ${ }^{a}$ & $p$-Value & $\begin{array}{c}\text { Low } \\
\text { Carbohydrates }{ }^{\text {a }}\end{array}$ & $\begin{array}{c}\text { High } \\
\text { Carbohydrates }{ }^{\text {a }}\end{array}$ & $p$-Value & Low Fat ${ }^{a}$ & High Fat ${ }^{a}$ & $p$-Value \\
\hline 14:0 & $10.23 \pm 9.48$ & $13.39 \pm 12.88$ & 0.213 & $12.01 \pm 11.49$ & $11.58 \pm 11.31$ & 0.924 & $11.28 \pm 10.86$ & $12.32 \pm 11.91$ & 0.812 \\
\hline 15:0 & $12.08 \pm 5.66$ & $12.15 \pm 5.03$ & 0.893 & $12.20 \pm 4.81$ & $12.02 \pm 5.86$ & 0.976 & $12.35 \pm 5.77$ & $11.88 \pm 4.89$ & 0.425 \\
\hline $16: 0$ & $417.76 \pm 148.18$ & $456.41 \pm 189.46$ & 0.316 & $440.62 \pm 175.05$ & $433.19 \pm 166.75$ & 0.902 & $426.90 \pm 157.45$ & $447.13 \pm 183.22$ & 0.694 \\
\hline 17:0 & $13.01 \pm 10.82$ & $15.66 \pm 9.53$ & 0.185 & $15.41 \pm 9.48$ & $13.23 \pm 10.94$ & 0.330 & $13.65 \pm 10.66$ & $15.01 \pm 9.84$ & 0.637 \\
\hline 18:0 & $273.15 \pm 55.14$ & $275.71 \pm 62.72$ & 0.973 & $273.11 \pm 59.65$ & $275.75 \pm 58.37$ & 0.640 & $272.05 \pm 58.44$ & $276.83 \pm 59.53$ & 0.896 \\
\hline 22:0 & $6.44 \pm 3.71$ & $6.45 \pm 3.51$ & 0.877 & $6.32 \pm 3.59$ & $6.57 \pm 3.63$ & 0.661 & $6.46 \pm 3.49$ & $6.42 \pm 3.73$ & 0.861 \\
\hline $14: 1 n-9$ & $4.58 \pm 2.71$ & $5.29 \pm 2.59$ & 0.254 & $5.02 \pm 2.50$ & $4.84 \pm 2.84$ & 0.909 & $4.94 \pm 2.91$ & $4.92 \pm 2.41$ & 0.762 \\
\hline $14: 1 n-5$ & $2.81 \pm 2.68$ & $2.32 \pm 1.72$ & 0.120 & $2.73 \pm 2.52$ & $2.40 \pm 1.96$ & 0.533 & $2.34 \pm 1.97$ & $2.79 \pm 2.51$ & 0.420 \\
\hline $15: 1$ & $27.66 \pm 11.10$ & $27.78 \pm 8.94$ & 0.895 & $26.74 \pm 8.24$ & $28.71 \pm 11.58$ & 0.213 & $27.95 \pm 11.04$ & $27.48 \pm 9.02$ & 0.887 \\
\hline $16: 1 n-7$ & $17.47 \pm 18.29$ & $27.17 \pm 26.81$ & 0.035 & $24.16 \pm 25.01$ & $20.37 \pm 21.54$ & 0.424 & $20.03 \pm 20.65$ & $24.57 \pm 25.74$ & 0.387 \\
\hline $17: 1$ & $34.47 \pm 19.87$ & $32.03 \pm 16.66$ & 0.567 & $29.61 \pm 14.93$ & $36.97 \pm 20.68$ & 0.020 & $33.97 \pm 19.49$ & $32.54 \pm 17.17$ & 0.914 \\
\hline $18: 1 n-9 t$ & $2.64 \pm 1.80$ & $2.67 \pm 1.73$ & 0.959 & $2.56 \pm 1.73$ & $2.75 \pm 1.79$ & 0.493 & $2.65 \pm 1.69$ & $2.66 \pm 1.84$ & 0.898 \\
\hline $18: 1 \mathrm{n}-7 \mathrm{t}$ & $4.79 \pm 3.14$ & $4.42 \pm 2.40$ & 0.448 & $4.62 \pm 2.73$ & $4.59 \pm 2.88$ & 0.950 & $4.53 \pm 3.07$ & $4.68 \pm 2.50$ & 0.799 \\
\hline $18: 1 n-9 c$ & $354.50 \pm 162.53$ & $403.01 \pm 209.02$ & 0.176 & $383.59 \pm 202.80$ & $373.47 \pm 172.90$ & 0.744 & $369.89 \pm 166.72$ & $387.38 \pm 208.17$ & 0.665 \\
\hline $18: 1 \mathrm{n}-7 \mathrm{c}$ & $27.54 \pm 10.52$ & $33.46 \pm 15.87$ & 0.022 & $31.53 \pm 14.88$ & $29.40 \pm 12.45$ & 0.457 & $29.27 \pm 12.79$ & $31.70 \pm 14.60$ & 0.403 \\
\hline $18: 1 n-5 c$ & $3.41 \pm 2.42$ & $5.26 \pm 4.28$ & 0.007 & $4.90 \pm 4.12$ & $3.75 \pm 2.85$ & 0.112 & $3.95 \pm 3.20$ & $4.72 \pm 3.91$ & 0.338 \\
\hline $20: 1 n-9$ & $7.09 \pm 2.88$ & $7.32 \pm 2.63$ & 0.621 & $7.33 \pm 2.80$ & $7.07 \pm 2.72$ & 0.674 & $7.26 \pm 2.71$ & $7.15 \pm 2.81$ & 0.723 \\
\hline $22: 1 n-9$ & $12.36 \pm 8.93$ & $10.98 \pm 8.03$ & 0.426 & $10.52 \pm 7.38$ & $12.86 \pm 9.39$ & 0.139 & $12.15 \pm 9.03$ & $11.20 \pm 7.94$ & 0.765 \\
\hline $24: 1 n-9$ & $13.89 \pm 12.59$ & $16.34 \pm 11.06$ & 0.237 & $15.34 \pm 10.97$ & $14.88 \pm 12.81$ & 0.946 & $14.09 \pm 12.33$ & $16.14 \pm 11.40$ & 0.312 \\
\hline $18: 2 n-6 t$ & $7.59 \pm 4.36$ & $10.47 \pm 4.55$ & 0.001 & $9.89 \pm 4.62$ & $8.14 \pm 4.58$ & 0.051 & $7.99 \pm 4.53$ & $10.07 \pm 4.60$ & 0.018 \\
\hline $18: 2 n-6$ & $263.82 \pm 161.35$ & $326.61 \pm 217.22$ & 0.092 & $312.01 \pm 207.61$ & $277.65 \pm 176.79$ & 0.333 & $276.75 \pm 175.44$ & $313.46 \pm 209.02$ & 0.381 \\
\hline $18: 3 n-6$ & $4.75 \pm 3.71$ & $6.45 \pm 5.19$ & 0.052 & $6.07 \pm 5.05$ & $5.11 \pm 4.00$ & 0.289 & $5.06 \pm 3.98$ & $6.13 \pm 5.07$ & 0.216 \\
\hline $20: 2 n-6$ & $4.69 \pm 8.70$ & $5.25 \pm 8.89$ & 0.641 & $5.29 \pm 8.85$ & $4.65 \pm 8.74$ & 0.634 & $5.67 \pm 10.16$ & $4.27 \pm 7.09$ & 0.405 \\
\hline $20: 3 n-6$ & $30.84 \pm 9.37$ & $33.49 \pm 12.71$ & 0.316 & $31.94 \pm 11.10$ & $32.37 \pm 11.37$ & 0.702 & $32.12 \pm 11.44$ & $32.19 \pm 11.02$ & 0.783 \\
\hline $20: 4 n-6$ & $278.58 \pm 55.85$ & $283.64 \pm 58.84$ & 0.939 & $282.06 \pm 59.92$ & $280.10 \pm 54.72$ & 0.804 & $280.99 \pm 57.55$ & $281.19 \pm 57.27$ & 0.534 \\
\hline $22: 2 n-6$ & $2.91 \pm 1.54$ & $3.00 \pm 1.71$ & 0.787 & $3.05 \pm 1.71$ & $2.86 \pm 1.53$ & 0.434 & $2.91 \pm 1.66$ & $3.00 \pm 1.60$ & 0.729 \\
\hline $22: 4 n-6$ & $47.91 \pm 12.16$ & $44.41 \pm 11.58$ & 0.068 & $44.45 \pm 11.80$ & $47.92 \pm 11.96$ & 0.060 & $48.33 \pm 12.03$ & $43.98 \pm 11.57$ & 0.019 \\
\hline $22: 5 n-6$ & $10.69 \pm 3.52$ & $10.81 \pm 3.34$ & 0.907 & $10.27 \pm 3.46$ & $11.24 \pm 3.33$ & 0.070 & $11.07 \pm 3.45$ & $10.44 \pm 3.39$ & 0.223 \\
\hline $18: 3 n-3 t$ & $2.33 \pm 2.33$ & $2.61 \pm 2.95$ & 0.672 & $2.41 \pm 2.35$ & $2.54 \pm 2.94$ & 0.695 & $2.16 \pm 2.24$ & $2.79 \pm 2.99$ & 0.177 \\
\hline $18: 3 n-3$ & $13.17 \pm 9.30$ & $14.16 \pm 8.18$ & 0.578 & $13.46 \pm 8.31$ & $13.86 \pm 9.21$ & 0.589 & $13.17 \pm 8.96$ & $14.15 \pm 8.55$ & 0.640 \\
\hline $20: 3 n-3$ & $9.06 \pm 8.32$ & $12.08 \pm 9.53$ & 0.072 & $11.46 \pm 9.71$ & $9.63 \pm 8.26$ & 0.353 & $9.95 \pm 8.61$ & $11.16 \pm 9.48$ & 0.539 \\
\hline $20: 5 n-3$ & $25.45 \pm 11.14$ & $27.74 \pm 14.05$ & 0.320 & $27.58 \pm 14.08$ & $25.57 \pm 11.09$ & 0.467 & $24.66 \pm 9.54$ & $28.54 \pm 15.04$ & 0.123 \\
\hline $22: 5 n-3$ & $134.26 \pm 61.66$ & $136.29 \pm 45.00$ & 0.990 & $142.04 \pm 50.02$ & $128.38 \pm 57.03$ & 0.193 & $133.53 \pm 53.37$ & $137.03 \pm 54.68$ & 0.986 \\
\hline $22: 6 n-3$ & $104.81 \pm 29.13$ & $97.72 \pm 26.87$ & 0.164 & $99.78 \pm 25.44$ & $102.83 \pm 30.79$ & 0.477 & $100.25 \pm 29.90$ & $102.35 \pm 26.44$ & 0.745 \\
\hline $20: 3 n-9$ & $5.50 \pm 2.18$ & $5.65 \pm 2.72$ & 0.682 & $5.55 \pm 2.69$ & $5.60 \pm 2.21$ & 0.928 & $5.38 \pm 2.03$ & $5.77 \pm 2.83$ & 0.254 \\
\hline
\end{tabular}

Values are means \pm standard deviations (SDs) for fatty acids levels. ${ }^{a}$ Low and high intakes determined by medians for proteins, carbohydrates, and fat, which were $16.85 \%$ energy intake, $54.86 \%$ energy intake, and $27.05 \%$ energy intake, respectively. The model was adjusted for body mass index (BMI), PA, and hypolipidemic and hypoglycemic medications. Abbreviations: SFA: saturated fatty acids; MUFA: monounsaturated fatty acids; $n-3$ PUFA: $n-3$ polyunsaturated fatty acids; $n-6$ PUFA: $n-6$ polyunsaturated fatty acids; RBC: red blood cells; BMI: body mass index; PA: physical activity. 
Table 4. Associations between FADS1 and FADS2 polymorphisms and fatty acid concentrations in erythrocyte membranes.

\begin{tabular}{|c|c|c|c|c|c|c|c|c|c|c|c|c|}
\hline \multirow{3}{*}{ Variable } & \multicolumn{12}{|c|}{ Polymorphisms } \\
\hline & \multicolumn{3}{|c|}{$\begin{array}{c}\text { rs174556 } \\
\text { FADS1 }\end{array}$} & \multicolumn{3}{|c|}{$\begin{array}{l}\text { rs174547 } \\
\text { FADS1 }\end{array}$} & \multicolumn{3}{|c|}{$\begin{array}{l}\text { rs174561 } \\
\text { FADS1 }\end{array}$} & \multicolumn{3}{|c|}{$\begin{array}{c}\text { rs3834458 } \\
\text { FADS2 }\end{array}$} \\
\hline & $\begin{array}{c}\text { CT + TT } \\
N=53\end{array}$ & $\begin{array}{c}\text { CC } \\
N=72\end{array}$ & $p$-Value & $\begin{array}{c}\text { TC }+ \text { CC } \\
N=54\end{array}$ & $\begin{array}{c}\text { TT } \\
N=72\end{array}$ & $p$-Value & $\begin{array}{c}\text { TC }+ \text { CC } \\
N=58\end{array}$ & $\begin{array}{c}\text { TT } \\
N=67\end{array}$ & $p$-Value & $\begin{array}{l}\mathrm{T} /-+-/- \\
N=59\end{array}$ & $\begin{array}{c}\text { TT } \\
N=66\end{array}$ & $p$-Value \\
\hline \multicolumn{13}{|c|}{$\mathrm{n}-6$ PUFA in RBC $(\mu \mathrm{g} / \mathrm{mL})$} \\
\hline $18: 2 n-6 t$ & $9.19 \pm 4.54$ & $8.85 \pm 4.84$ & NS & $9.24 \pm 4.51$ & $8.85 \pm 4.84$ & NS & $8.90 \pm 4.38$ & $9.08 \pm 4.99$ & NS & $8.90 \pm 4.58$ & $9.08 \pm 4.83$ & NS \\
\hline $18: 2 n-6$ & $312.16 \pm 202.00$ & $286.07 \pm 188.05$ & NS & $309.59 \pm 200.98$ & $286.07 \pm 188.05$ & NS & $293.42 \pm 192.49$ & $300.35 \pm 196.16$ & NS & $304.90 \pm 193.68$ & $290.19 \pm 194.97$ & NS \\
\hline $18: 3 \mathrm{n}-6$ & $5.60 \pm 3.80$ & $5.67 \pm 5.12$ & NS & $5.54 \pm 3.79$ & $5.67 \pm 5.12$ & NS & $5.55 \pm 3.77$ & $5.72 \pm 5.22$ & NS & $5.61 \pm 3.87$ & $5.67 \pm 5.18$ & NS \\
\hline $20: 2 \mathrm{n}-6$ & $4.40 \pm 6.85$ & $5.53 \pm 10.04$ & NS & $4.32 \pm 6.81$ & $5.53 \pm 10.04$ & NS & $4.39 \pm 6.61$ & $5.62 \pm 10.37$ & NS & $4.19 \pm 6.54$ & $5.81 \pm 10.44$ & NS \\
\hline $20: 3 n-6$ & $34.06 \pm 11.04$ & $30.81 \pm 11.32$ & NS & $34.05 \pm 10.94$ & $30.81 \pm 11.32$ & NS & $32.97 \pm 10.85$ & $31.50 \pm 11.66$ & NS & $33.63 \pm 11.06$ & $30.90 \pm 11.39$ & NS \\
\hline $20: 4 n-6$ & $267.92 \pm 55.96$ & $289.97 \pm 57.23$ & NS & $268.81 \pm 55.81$ & $289.97 \pm 57.23$ & NS & $265.27 \pm 54.84$ & $293.90 \pm 56.85$ & $<0.05$ & $270.18 \pm 54.81$ & $289.95 \pm 58.67$ & NS \\
\hline $22: 2 \mathrm{n}-6$ & $2.96 \pm 1.87$ & $2.96 \pm 1.45$ & NS & $2.96 \pm 1.86$ & $2.96 \pm 1.45$ & NS & $2.90 \pm 1.80$ & $3.02 \pm 1.49$ & NS & $3.02 \pm 1.91$ & $2.91 \pm 1.36$ & NS \\
\hline $22: 4 n-6$ & $44.97 \pm 13.65$ & $47.10 \pm 10.70$ & NS & $45.07 \pm 13.54$ & $47.10 \pm 10.70$ & NS & $45.01 \pm 12.96$ & $47.22 \pm 11.17$ & NS & $45.35 \pm 13.67$ & $46.95 \pm 10.41$ & NS \\
\hline $22: 5 n-6$ & $11.06 \pm 3.67$ & $10.57 \pm 3.27$ & NS & $11.03 \pm 3.65$ & $10.57 \pm 3.27$ & NS & $10.91 \pm 3.41$ & $10.67 \pm 3.49$ & NS & $11.01 \pm 3.57$ & $10.58 \pm 3.34$ & NS \\
\hline \multicolumn{13}{|c|}{$\mathrm{n}-3$ PUFA in RBC $(\mu \mathrm{g} / \mathrm{mL})$} \\
\hline $18: 3 n-3 t$ & $2.22 \pm 1.93$ & $2.70 \pm 3.09$ & NS & $2.20 \pm 1.92$ & $2.70 \pm 3.09$ & NS & $2.13 \pm 1.44$ & $2.82 \pm 3.37$ & NS & $2.23 \pm 1.89$ & $2.74 \pm 3.20$ & NS \\
\hline $18: 3 n-3$ & $12.96 \pm 7.10$ & $14.33 \pm 9.84$ & NS & $12.84 \pm 7.09$ & $14.33 \pm 9.84$ & NS & $12.40 \pm 6.65$ & $14.92 \pm 10.18$ & NS & $12.72 \pm 7.17$ & $14.67 \pm 9.97$ & NS \\
\hline $20: 3 n-3$ & $10.62 \pm 8.29$ & $10.74 \pm 9.62$ & NS & $10.47 \pm 8.28$ & $10.74 \pm 9.62$ & NS & $10.03 \pm 7.73$ & $11.26 \pm 10.07$ & NS & $11.02 \pm 9.30$ & $10.40 \pm 8.87$ & NS \\
\hline $20: 5 n-3$ & $24.89 \pm 12.80$ & $27.97 \pm 12.64$ & NS & $24.75 \pm 12.72$ & $27.97 \pm 12.64$ & NS & $24.42 \pm 12.79$ & $28.61 \pm 12.48$ & NS & $24.73 \pm 12.56$ & $28.40 \pm 12.76$ & NS \\
\hline $22: 5 n-3$ & $129.50 \pm 54.13$ & $138.00 \pm 53.68$ & NS & $130.53 \pm 54.16$ & $138.00 \pm 53.68$ & NS & $128.93 \pm 54.39$ & $139.13 \pm 53.27$ & NS & $132.32 \pm 53.54$ & $136.26 \pm 54.40$ & NS \\
\hline $22: 6 \mathrm{n}-3$ & $101.03 \pm 29.62$ & $101.13 \pm 27.15$ & NS & $100.76 \pm 29.40$ & $101.13 \pm 27.15$ & NS & $100.21 \pm 29.35$ & $101.85 \pm 27.18$ & NS & $101.01 \pm 28.77$ & $101.16 \pm 27.72$ & NS \\
\hline
\end{tabular}

Values are means \pm SDs for fatty acids levels. The model was adjusted for BMI, PA, and hypolipidemic and hypoglycemic medications. Abbreviations: SFA: saturated fatty acids; MUFA: monounsaturated fatty acids; n-3 PUFA: n-3 polyunsaturated fatty acids; n-6 PUFA: n-6 polyunsaturated fatty acids; RBC: Red blood cells; BMI: Body mass index; PA: Physical activity, NS: not significant.

Table 5. Desaturase and elongase index characteristics.

\begin{tabular}{cccc}
\hline Parameter & Mean \pm SD & Minimum & Maximum \\
\hline \multicolumn{4}{c}{ Desaturase activity ratios } \\
\hline D5D & $9.40 \pm 0.21$ & 4.95 & 15.37 \\
D6D & $0.021 \pm 0.001$ & 0.003 & 0.057 \\
\hline \multicolumn{4}{c}{ Combined effect of desaturase and elongase activity ratios } \\
\hline 20:4n-6/18:2n-6 & $1.24 \pm 0.05$ & 0.32 & 2.34 \\
$22: 6 \mathrm{n}-3 / 20: 5 \mathrm{n}-3$ & $4.27 \pm 0.12$ & 1.22 & 8.98 \\
$20: 5 \mathrm{n}-3 / 18: 3 \mathrm{n}-3$ & $2.41 \pm 0.12$ & 0.48 & 8.40 \\
$22: 6 \mathrm{n}-3 / 18: 3 \mathrm{n}-3$ & $10.16 \pm 0.52$ & 2.12 & 27.38 \\
22:4n-6/18:2n-6 & $0.22 \pm 0.01$ & 0.03 & 0.50 \\
\hline
\end{tabular}

$N=130$; abbreviations: D5D: Delta-5 desaturase index, 20:4n-6/20:3n-6; D6D: delta-6 desaturase index, 18:3n-6/18:2n-6. 
Table 6. Associations between FADS1 and FADS2 polymorphisms and desaturase and elongase indexes.

\begin{tabular}{|c|c|c|c|c|c|c|c|c|c|c|c|c|}
\hline \multirow{3}{*}{ Variable } & \multicolumn{12}{|c|}{ Polymorphisms } \\
\hline & \multicolumn{3}{|c|}{$\begin{array}{c}\text { rs174556 } \\
\text { FADS1 }\end{array}$} & \multicolumn{3}{|c|}{$\begin{array}{c}\text { rs174547 } \\
\text { FADS1 }\end{array}$} & \multicolumn{3}{|c|}{$\begin{array}{c}\text { rs174561 } \\
\text { FADS1 }\end{array}$} & \multicolumn{3}{|c|}{$\begin{array}{c}\text { rs3834458 } \\
\text { FADS2 }\end{array}$} \\
\hline & $\begin{array}{c}\mathrm{CT}+\mathrm{TT} \\
N=53\end{array}$ & $\begin{array}{c}\mathrm{CC} \\
N=72\end{array}$ & $p$-Value & $\begin{array}{c}\mathrm{TC}+\mathrm{CC} \\
N=54\end{array}$ & $\begin{array}{c}\text { TT } \\
N=72\end{array}$ & $p$-Value & $\begin{array}{c}\mathrm{TC}+\mathrm{CCN} \\
N=58\end{array}$ & $\begin{array}{c}\text { TT } \\
N=67\end{array}$ & $p$-Value & $\begin{array}{l}\mathrm{T} /-+-/- \\
N=59\end{array}$ & $\begin{array}{c}\text { TT } \\
N=66\end{array}$ & $p$-Value \\
\hline \multicolumn{13}{|c|}{ Desaturase activity ratios } \\
\hline D5D & $8.29 \pm 1.97$ & $10.10 \pm 2.37$ & $<0.0001$ & $8.31 \pm 1.96$ & $10.10 \pm 2.37$ & $<0.0001$ & $8.51 \pm 2.12$ & $10.04 \pm 2.37$ & $<0.001$ & $8.52 \pm 2.14$ & $10.06 \pm 2.36$ & $<0.001$ \\
\hline D6D & $0.02 \pm 0.01$ & $0.02 \pm 0.01$ & NS & $0.02 \pm 0.01$ & $0.02 \pm 0.01$ & NS & $0.02 \pm 0.01$ & $0.02 \pm 0.01$ & NS & $0.02 \pm 0.01$ & $0.02 \pm 0.01$ & NS \\
\hline \multicolumn{13}{|c|}{ Combined effect of desaturase and elongase activity ratios } \\
\hline $20: 4 n-6 / 18: 2 n-6$ & $1.14 \pm 0.52$ & $1.30 \pm 0.50$ & $<0.05$ & $1.15 \pm 0.52$ & $1.30 \pm 0.50$ & NS & $1.18 \pm 0.50$ & $1.27 \pm 0.52$ & NS & $1.16 \pm 0.50$ & $1.30 \pm 0.51$ & NS \\
\hline $22: 4 n-6 / 18: 2 n-6$ & $0.21 \pm 0.12$ & $0.23 \pm 0.12$ & NS & $0.21 \pm 0.12$ & $0.23 \pm 0.12$ & NS & $0.22 \pm 0.12$ & $0.22 \pm 0.12$ & NS & $0.21 \pm 0.12$ & $0.23 \pm 0.12$ & NS \\
\hline $20: 5 n-3 / 18: 3 n-3$ & $2.29 \pm 1.28$ & $2.52 \pm 1.43$ & NS & $2.29 \pm 1.27$ & $2.52 \pm 1.43$ & NS & $2.30 \pm 1.25$ & $2.52 \pm 1.47$ & NS & $2.33 \pm 1.26$ & $2.50 \pm 1.47$ & NS \\
\hline $22: 6 n-3 / 18: 3 n-3$ & $10.01 \pm 5.61$ & $10.22 \pm 6.39$ & NS & $10.06 \pm 5.57$ & $10.22 \pm 6.39$ & NS & $10.33 \pm 5.80$ & $9.95 \pm 6.29$ & NS & $10.37 \pm 5.84$ & $9.91 \pm 6.26$ & NS \\
\hline $22: 6 n-3 / 20: 5 n-3$ & $4.49 \pm 1.34$ & $4.04 \pm 1.28$ & NS & $4.50 \pm 1.33$ & $4.04 \pm 1.28$ & NS & $4.57 \pm 1.36$ & $3.95 \pm 1.23$ & $<0.05$ & $4.53 \pm 1.36$ & $3.97 \pm 1.24$ & $<0.05$ \\
\hline
\end{tabular}

Values are means \pm standard deviations (SDs) for fatty acids levels. The model was adjusted for age, body mass, physical activity, hypolipidemic and hypoglycemic medications, and intake of enzyme precursors. Abbreviations: D5D: delta-5 desaturase index, 20:4n-6/20:3n-6; D6D: delta-6 desaturase index, 18:3n-6/18:2n-6. 


\section{Discussion}

To the best of our knowledge, this is the first study to investigate the relationship between FA composition in erythrocyte membranes, FADS1 and FADS2 gene polymorphism, and macronutrient intake (specifically FA intake) in centrally obese postmenopausal Polish women.

Generally, we saw only a few statistically significant associations between the amounts of protein, fat, and carbohydrates in the diet and the FA profile in erythrocyte membranes (Table 3). We observed that high protein intake and low carbohydrate intake were associated with higher levels of some examined FAs in RBC. There have been no studies of the associations between macronutrient intakes and FA profile, but Novak and Innis (2012) found that protein intake and EPA and DHA intakes were positively correlated, while there was no such correlation with fat intake [41]. For this reason, we anticipated that protein intake might affect the concentrations of EPA, DHA, and other FAs in RBC. Although these particular associations were not observed in our study, the results overall confirm previous suggestions that recommendations for EPA and DHA intake should focus on protein sources, rather than on dietary fat [41,42]. Generally, guidelines for consumers have acknowledged the link between protein sources and suggest weekly numbers of fish servings to provide EPA and DHA [43]. It should be underlined, however, that in populations where seafood intake is low, as in our study, animal-derived foods are important sources of fatty acids. Most likely the observed associations between protein intake and particular fatty acids concentrations (16:1n-7, 18:1n-9c, 18:2n-6 or 18:1n-7c) result from the diet structure characteristic of Poland, where dairy and poultry products are often the main protein sources [44,45]. In our study, around $65 \%$ of proteins were derived from products of animal origin. Moreover, pork and poultry provided over $30 \%$ of the total protein intake, while milk and dairy products made up more than $20 \%$ of the total protein intake. Fish were consumed less frequently and provided around $4 \%$ of total protein (data not shown). Interestingly, we also observed that protein intake was associated with the 22:6n-3/20:5n-3 and 22:6n-3/22:5n-3 ratios, and that the values of these indices were lower in women who met their needs for protein $(1.2 \mathrm{~g}$ of protein $/ \mathrm{kg}$ of body mass/day). It is also worth mentioning that only concentrations of $18: 2 n-6 \mathrm{t}$ in RBC were associated with the intake of all macronutrients-higher 18:2n-6t levels in RBC were associated with higher total protein, or fat intake, or with lower carbohydrate intake. Trans-FAs are provided by high protein and high-fat products and are formed by technological processes [46]. Together, these results show that dietary habits related to protein intake may affect the FA profile in RBC, and increased fish intake is advisable in Polish postmenopausal women. This is of great importance for this age group, as the adequate intake of food items that are sources of n-3 LC-PUFA can lead to an increase in n-3 LC-PUFA in the RBC, an improvement in plasma lipid profile, and a reduction in cardiovascular risk factors and inflammatory mediators [47].

Furthermore, we examined the relationships between FA intake and composition in erythrocyte membranes. Associations were observed between high SFA intake and high 18:2n-6t, and 17:0 and low 22:4n-6 in RBC (Table S1), and also between high MUFA intake and low 22:5n-6 in RBC. Other studies have described several associations between SFA and unSFA from diet and FA status in the human sample [48-55] and, as mentioned above, FA status may significantly contribute to metabolic health. It has been shown that high levels of total SFA, low levels of PUFA, and high levels of different FAs in human plasma or RBC (mainly, 16:0, 16:1n-7, 18:0, n-6 (18:3n-6, 20:3n-6, AA), and EPA) are associated with the risk of obesity or MetS [56-60]. That we did not find some of the associations often found in other studies might be due to age, sex, postmenopausal status, or central obesity in our study group. The differences could also be explained by the fact that, in our study, we examined FAs in RBC, while the other studies studied FA in serum and plasma (or plasma phospholipids).

The n-3 and n-6 PUFA pathways compete with each other for desaturases and elongases. High levels of dietary precursors (ALA and LA) in human samples could reflect high dietary intake of these FA, or diminished conversion to LC-PUFA. D5D, encoded by FADS1, is involved in the conversion of 20:4n-3 to 20:5n-3 and of 20:3n-6 to 20:4n-6 [61,62], and FADS1 genotype may be associated with metabolic efficiency. In our study, rs174561 polymorphism of the FADS1 gene was associated with 
higher 20:4n-6 concentration in RBC in the major allele carriers $(p<0.05)$, but there were no other associations between other FAs in RBC levels and FADS1 or FADS2 polymorphisms (Table 4). This is consistent with the results of several previous studies [11-13,22,63], where the minor allele carriers of rs174556, rs174547, rs174561, and rs3834458 had higher concentrations of 18:2n-6, 20:3n-6, 18:3n-3 and lower concentrations of 18:3n-6, 20:4n-6, 22:4n-6, 20:5n-3, and 22:5n-3 in serum, plasma phospholipids or RBC. A high level of AA and a low level of EPA, as well as a high AA/EPA ratio, are associated with cardiovascular disorders [64]. AA is a precursor in the conversion of proinflammatory compounds, such as prostaglandins and leukotrienes, so higher levels of AA in the major allele carriers could result in increased inflammation $[65,66]$. Moreover, in contrast to these studies, in our study, subjects with at least one minor allele of rs174561 had lower levels of 18:2n-6 (Table 4).

In our study, desaturase and elongase activities were estimated using product-to-substrate ratios. The values of D6D obtained were comparable to those observed in other studies with healthy subjects, but the D5D values were higher in our study [52,67]. High D5D activity is associated with lower risk of cardiovascular incidences and also with good insulin sensitivity $[52,65]$ while a low D6D activity index is associated with a lower risk of mortality, insulin resistance, and obesity $[52,57,65,68]$. The mean D5D and D6D values observed in our postmenopausal centrally obese study sample thus seem surprising, and the reasons for this are unknown. It has been indicated that diet, environmental exposure, and life stage can potentially affect elongase and desaturase activity [1]. Aging may decrease D6D activity in rodents and humans. Decreased activity of D6D with age may not apply to different cohorts, as either males or mixed gender samples have usually been analyzed [69]. Studies of aging women were unable to document a similar decline in enzyme activity, primarily because of the released suppression of desaturase activity with the loss of estrogen after menopause. Estrogen is known to suppress fatty acid desaturation in cell culture and rats [69]. It was also noted that insulin, though not estrogen, appears to influence desaturase activity, as demonstrated by the increased desaturase function associated with hyperinsulinemia in obese women [70]. It should, however, be underlined that desaturase indices, calculated as product-to-substrate ratios, are only an indirect measure of desaturase activity.

We additionally observed that the mean D5D value was significantly lower in the minor allele carriers of rs174556, rs174561, and rs174547 of FADS1 and of rs3834458 of the FADS2 gene. There have been only a few studies to examine the associations between FADS genotype and desaturase and elongase activity in women but, to the best of our knowledge, there has been no study on this topic in postmenopausal women; this limits interpretation [71-74]. However, our results are consistent with previous findings of decreased desaturase activities in minor allele carriers [23].

As shown in our study and several previous studies, FA metabolism depends on diet and genetically determined desaturase activities. These studies have practical implications: In particular, people with unfavorable genotypes and higher intake of n-3 FAs-mainly of 18:3n-3, DHA, and EPA-might be advised to reduce the risk of inflammation or MetS.

One strength of the presented study is that FA composition was analyzed in erythrocyte membranes, which reflects the long-term FA intake and it is known as the best noninvasive biomarker of FA intake in humans. Moreover, the study group was well defined and homogenous, leading to low interindividual variability. One weakness of this study is the small sample size. Moreover, the product-to-substrate estimation, a traditional approach, was used to assess enzymatic activity, but no protein activity was determined. Another limitation could be that we did not include all the potential confounders in the multiple regression analysis. These include, for example, data on liver functioning. Further, to assess habitual intake of FAs we did not use a retrospective method. It is however worth mentioning that our study presents for the first time the relationship between macronutrients and FA intake and genotype and FA composition in erythrocyte membranes in postmenopausal Polish women.

\section{Conclusions}

We conclude that protein or carbohydrate intake may be associated with FA concentration in the RBC in centrally obese postmenopausal Polish women. Moreover, SFA, MUFA, or PUFA may not be 
associated with FA levels in RBC in this study group. The potential implications of these findings should be investigated in a further intervention study considering non obese population. The D5D value, though not the D6D value, may be affected by FADS1 or FADS2 polymorphism.

Supplementary Materials: The following are available online at http:/ /www.mdpi.com/2072-6643/10/8/1068/ s1, Table S1: Distribution of erythrocyte fatty acids by median total SFA intake, Table S2: Distribution of erythrocyte fatty acids by median total MUFA intake, Table S3: Distribution of erythrocyte fatty acids by median total PUFA intake.

Author Contributions: Conceptualization, A.M., A.C.; Methodology, A.M., A.G., H.H.J., J.B., A.C.; Validation, A.M., H.H.J., J.B., A.C.; Formal Analysis, A.C.; Investigation, A.M., J.B., A.C.; Resources, A.M., A.C.; Data Curation, A.M., A.C.; Writing-Original Draft Preparation, A.M., A.C.; Writing-Review \& Editing, A.M., H.H.J., J.B., A.C.; Visualization, A.M.; Supervision, A.C.; Project Administration, A.M.; Funding Acquisition, A.M., J.B., A.C.

Funding: The study was partly financed by a National Science Centre award, based on decision number DEC-013/09/B/NZ9/02365 and also the Ministry of Science and Higher Education, funding for young scientists.

Conflicts of Interest: The authors declare no conflicts of interest. The founding sponsors had no role in the design of the study, in the collection, analysis, or interpretation of the data, in the writing of the manuscript, or in the decision to publish the results.

\section{References}

1. Murff, H.J.; Edwards, T.L. Endogenous Production of Long-Chain Polyunsaturated Fatty Acids and Metabolic Disease Risk. Curr. Cardiovasc. Risk Rep. 2014, 8, 418. [CrossRef] [PubMed]

2. Dubnov, G.; Brzezinski, A.; Berry, E.M. Weight control and the management of obesity after menopause: The role of physical activity. Maturitas 2003, 44, 89-101. [CrossRef]

3. Lobo, R.A. Metabolic syndrome after menopause and the role of hormones. Maturitas 2008, 60, 10-18. [CrossRef] [PubMed]

4. Mastorakos, G.; Valsamakis, G.; Paltoglou, G.; Creatsas, G. Management of obesity in menopause: Diet, exercise, pharmacotherapy and bariatric surgery. Maturitas 2010, 65, 219-224. [CrossRef] [PubMed]

5. Vryonidou, A.; Paschou, S.A.; Muscogiuri, G.; Orio, F.; Goulis, D.G. Mechanisms in endocrinology: Metabolic syndrome through the female life cycle. Eur. J. Endocrinol. 2015, 173, R153-R163. [CrossRef] [PubMed]

6. Rautiainen, S.; Wang, L.; Lee, I.-M.; Manson, J.E.; Buring, J.E.; Sesso, H.D. Higher Intake of Fruit, but Not Vegetables or Fiber, at Baseline Is Associated with Lower Risk of Becoming Overweight or Obese in Middle-Aged and Older Women of Normal BMI at Baseline. J. Nutr. 2015, 145, 960-968. [CrossRef] [PubMed]

7. De Almeida Ventura, D.; de Matos Fonseca, V.; Ramos, E.G.; Marinheiro, L.P.F.; de Souza, R.A.G.; de Miranda Chaves, C.R.M.; Peixoto, M.V.M. Association between quality of the diet and cardiometabolic risk factors in postmenopausal women. Nutr. J. 2014, 13, 121. [CrossRef] [PubMed]

8. Tardivo, A.P.; Nahas-Neto, J.; Nahas, E.A.P.; Maesta, N.; Rodrigues, M.A.; Orsatti, F.L. Associations between healthy eating patterns and indicators of metabolic risk in postmenopausal women. Nutr. J. 2010, 9, 64. [CrossRef] [PubMed]

9. Grygiel-Górniak, B.; Marcinkowska, J.; Szczepanik, A.; Przysławski, J. Nutritional habits and oxidative stress in postmenopausal age. Pol. Arch. Med. Wewn. 2014, 124, 298-305. [CrossRef] [PubMed]

10. Grygiel-Górniak, B.; Kaczmarek, E.; Mosor, M.; Przysławski, J.; Nowak, J. The gene-diet associations in postmenopausal women with newly diagnosed dyslipidemia. J. Nutr. Heal. Aging 2017, 21, 1031-1037. [CrossRef] [PubMed]

11. Schaeffer, L.; Gohlke, H.; Müller, M.; Heid, I.M.; Palmer, L.J.; Kompauer, I.; Demmelmair, H.; Illig, T.; Koletzko, B.; Heinrich, J. Common genetic variants of the FADS1 FADS2 gene cluster and their reconstructed haplotypes are associated with the fatty acid composition in phospholipids. Hum. Mol. Genet. 2006, 15, 1745-1756. [CrossRef] [PubMed]

12. Rzehak, P.; Heinrich, J.; Klopp, N.; Schaeffer, L.; Hoff, S.; Wolfram, G.; Illig, T.; Linseisen, J. Evidence for an association between genetic variants of the fatty acid desaturase 1 fatty acid desaturase 2 (FADS1 FADS2) gene cluster and the fatty acid composition of erythrocyte membranes. Br. J. Nutr. 2009, 101, 20. [CrossRef] [PubMed] 
13. Malerba, G.; Schaeffer, L.; Xumerle, L.; Klopp, N.; Trabetti, E.; Biscuola, M.; Cavallari, U.; Galavotti, R.; Martinelli, N.; Guarini, P.; et al. SNPs of the FADS Gene Cluster are Associated with Polyunsaturated Fatty Acids in a Cohort of Patients with Cardiovascular Disease. Lipids 2008, 43, 289-299. [CrossRef] [PubMed]

14. Zietemann, V.; Kröger, J.; Enzenbach, C.; Jansen, E.; Fritsche, A.; Weikert, C.; Boeing, H.; Schulze, M.B. Genetic variation of the FADS1 FADS2 gene cluster and n-6 PUFA composition in erythrocyte membranes in the European Prospective Investigation into Cancer and Nutrition-Potsdam study. Br. J. Nutr. 2018, 104, 1748-1759. [CrossRef] [PubMed]

15. Chilton, F.; Murphy, R.; Wilson, B.; Sergeant, S.; Ainsworth, H.; Seeds, M.; Mathias, R. Diet-Gene Interactions and PUFA Metabolism: A Potential Contributor to Health Disparities and Human Diseases. Nutrients 2014, 6, 1993-2022. [CrossRef] [PubMed]

16. Minihane, A.M. Impact of Genotype on EPA and DHA Status and Responsiveness to Increased Intakes. Nutrients 2016, 8, 123. [CrossRef] [PubMed]

17. Chilton, F.H.; Dutta, R.; Reynolds, L.M.; Sergeant, S.; Mathias, R.A.; Seeds, M.C. Precision nutrition and omega-3 polyunsaturated fatty acids: A case for personalized supplementation approaches for the prevention and management of human diseases. Nutrients 2017, 9, 1165. [CrossRef] [PubMed]

18. Mathias, R.A.; Pani, V.; Chilton, F.H. Genetic Variants in the FADS Gene: Implications for Dietary Recommendations for Fatty Acid Intake. Curr. Nutr. Rep. 2014, 3, 139-148. [CrossRef] [PubMed]

19. Hallmann, J.; Kolossa, S.; Gedrich, K.; Celis-Morales, C.; Forster, H.; O’Donovan, C.B.; Woolhead, C.; Macready, A.L.; Fallaize, R.; Marsaux, C.F.M.; et al. Predicting fatty acid profiles in blood based on food intake and the FADS1 rs174546 SNP. Mol. Nutr. Food Res. 2015, 59, 2565-2573. [CrossRef] [PubMed]

20. Baylin, A.; Ruiz-Narvaez, E.; Kraft, P.; Campos, H. $\alpha$-Linolenic acid, $\Delta 6$-desaturase gene polymorphism, and the risk of nonfatal myocardial infarction. Am. J. Clin. Nutr. 2007, 85, 554-560. [CrossRef] [PubMed]

21. Illig, T.; Gieger, C.; Zhai, G.; Römisch-Margl, W.; Wang-Sattler, R.; Prehn, C.; Altmaier, E.; Kastenmüller, G.; Kato, B.S.; Mewes, H.-W.; et al. A genome-wide perspective of genetic variation in human metabolism. Nat. Genet. 2010, 42, 137-141. [CrossRef] [PubMed]

22. Moltó-Puigmartí, C.; Plat, J.; Mensink, R.P.; Müller, A.; Jansen, E.; Zeegers, M.P.; Thijs, C. FADS1 FADS2 gene variants modify the association between fish intake and the docosahexaenoic acid proportions in human milk. Am. J. Clin. Nutr. 2010, 91, 1368-1376. [CrossRef] [PubMed]

23. Bokor, S.; Dumont, J.; Spinneker, A.; Gonzalez-Gross, M.; Nova, E.; Widhalm, K.; Moschonis, G.; Stehle, P.; Amouyel, P.; De Henauw, S.; et al. Single nucleotide polymorphisms in the FADS gene cluster are associated with delta- 5 and delta- 6 desaturase activities estimated by serum fatty acid ratios. J. Lipid Res. 2010, 51, 2325-2333. [CrossRef] [PubMed]

24. Rzehak, P.; Thijs, C.; Standl, M.; Mommers, M.; Glaser, C.; Jansen, E.; Klopp, N.; Koppelman, G.H.; Singmann, P.; Postma, D.S.; et al. LISA study group Variants of the FADS1 FADS2 gene cluster, blood levels of polyunsaturated fatty acids and eczema in children within the first 2 years of life. PLOS ONE 2010, 5, e13261. [CrossRef] [PubMed]

25. Koletzko, B.; Lattka, E.; Zeilinger, S.; Illig, T.; Steer, C. Genetic variants of the fatty acid desaturase gene cluster predict amounts of red blood cell docosahexaenoic and other polyunsaturated fatty acids in pregnant women: Findings from the Avon Longitudinal Study of Parents and Children. Am. J. Clin. Nutr. 2011, 93, 211-219. [CrossRef] [PubMed]

26. Lattka, E.; Rzehak, P.; Szabó, É.; Jakobik, V.; Weck, M.; Weyermann, M.; Grallert, H.; Rothenbacher, D.; Heinrich, J.; Brenner, H.; et al. Genetic variants in the FADS gene cluster are associated with arachidonic acid concentrations of human breast milk at 1.5 and 6 mo postpartum and influence the course of milk dodecanoic, tetracosenoic, and trans-9-octadecenoic acid concentrations over the duration of lactation. Am. J. Clin. Nutr. 2011, 93, 382-391. [CrossRef] [PubMed]

27. Sergeant, S.; Hugenschmidt, C.E.; Rudock, M.E.; Ziegler, J.T.; Ivester, P.; Ainsworth, H.C.; Vaidya, D.; Douglas Case, L.; Langefeld, C.D.; Freedman, B.I.; et al. Differences in arachidonic acid levels and fatty acid desaturase (FADS) gene variants in African Americans and European Americans with diabetes or the metabolic syndrome. Br. J. Nutr. 2012, 107, 547-555. [CrossRef] [PubMed]

28. Xie, L.; Innis, S.M. Genetic Variants of the FADS1 FADS2 Gene Cluster Are Associated with Altered (n-6) and (n-3) Essential Fatty Acids in Plasma and Erythrocyte Phospholipids in Women during Pregnancy and in Breast Milk during Lactation. J. Nutr. 2008, 138, 2222-2228. [CrossRef] [PubMed] 
29. Horiguchi, S.; Nakayama, K.; Iwamoto, S.; Ishijima, A.; Minezaki, T.; Baba, M.; Kontai, Y.; Horikawa, C.; Kawashima, H.; Shibata, H.; et al. Associations between a fatty acid desaturase gene polymorphism and blood arachidonic acid compositions in Japanese elderly. Prostaglandins Leukot. Essent. Fat. Acids 2015, 105, 9-14. [CrossRef] [PubMed]

30. Martinelli, N.; Girelli, D.; Malerba, G.; Guarini, P.; Illig, T.; Trabetti, E.; Sandri, M.; Friso, S.; Pizzolo, F.; Schaeffer, L.; et al. FADS genotypes and desaturase activity estimated by the ratio of arachidonic acid to linoleic acid are associated with inflammation and coronary artery disease. Am. J. Clin. Nutr. 2008, 88, 941-949. [CrossRef] [PubMed]

31. Mathias, R.A.; Vergara, C.; Gao, L.; Rafaels, N.; Hand, T.; Campbell, M.; Bickel, C.; Ivester, P.; Sergeant, S.; Barnes, K.C.; et al. FADS genetic variants and $\omega-6$ polyunsaturated fatty acid metabolism in a homogeneous island population. J. Lipid Res. 2010, 51, 2766. [CrossRef] [PubMed]

32. Schuchardt, J.P.; Köbe, T.; Witte, V.; Willers, J.; Gingrich, A.; Tesky, V.; Pantel, J.; Rujescu, D.; Illig, T.; Flöel, A.; et al. Genetic variants of the FADS gene cluster are associated with erythrocyte membrane LC PUFA levels in patients with mild cognitive impairment. J. Nutr. Heal. Aging 2016, 20, 611-620. [CrossRef] [PubMed]

33. Bajerska, J.; Chmurzynska, A.; Muzsik, A.; Krzyżanowska, P.; Mądry, E.; Malinowska, A.M.; Walkowiak, J. Weight loss and metabolic health effects from energy-restricted Mediterranean and Central-European diets in postmenopausal women: A randomized controlled trial. Sci. Rep. 2018, 8, 11170. [CrossRef] [PubMed]

34. Ortega, R.M.; Pérez-Rodrigo, C.; López-Sobaler, A.M. Dietary assessment methods: Dietary records. Nutr. Hosp. 2015, 31, 38-45. [CrossRef] [PubMed]

35. Jarosz, M. Nutrition Standards for the Polish Population-Amendment (Report in Polish). Warsaw. 2017. Available online: http:/ / www.izz.waw.pl/pl/normy-zwienia (accessed on 20 January 2018).

36. Zimmet, P.; Magliano, D.; Matsuzawa, Y.; Alberti, G.; Shaw, J. The metabolic syndrome: A global public health problem and a new definition. J. Atheroscler. Thromb. 2005, 12, 295-300. [CrossRef] [PubMed]

37. Craig, C.L.; Marshall, A.L.; Sjöström, M.; Bauman, A.E.; Booth, M.L.; Ainsworth, B.E.; Pratt, M.; Ekelund, U.; Yngve, A.; Sallis, J.F.; et al. International Physical Activity Questionnaire: 12-Country Reliability and Validity. Med. Sci. Sport. Exerc. 2003, 35, 1381-1395. [CrossRef] [PubMed]

38. Folch, J.; Lees, M.; Sloane Stanley, G.H. A simple method for the isolation and purification of total lipides from animal tissues. J. Biol. Chem. 1957, 226, 497-509. [PubMed]

39. Jacobs, S.; Jäger, S.; Jansen, E.; Peter, A.; Stefan, N.; Boeing, H.; Schulze, M.B.; Kröger, J. Associations of Erythrocyte Fatty Acids in the De Novo Lipogenesis Pathway with Proxies of Liver Fat Accumulation in the EPIC-Potsdam Study. PLoS ONE 2015, 10, e0127368. [CrossRef] [PubMed]

40. Paglialunga, S.; Dehn, C.A. Clinical assessment of hepatic de novo lipogenesis in non-alcoholic fatty liver disease. Lipids Health Dis. 2016, 15, 159. [CrossRef] [PubMed]

41. Novak, E.M.; Innis, S.M. Dietary long chain n-3 fatty acids are more closely associated with protein than energy intakes from fat. Prostaglandins Leukot. Essent. Fat. Acids 2012, 86, 107-112. [CrossRef] [PubMed]

42. Stark, K.D.; Patterson, A.C. EPA and DHA-Protein, not fat is "Where It's at"? Prostaglandins Leukot. Essent. Fat. Acids 2012, 87, 49-51. [CrossRef] [PubMed]

43. Richter, C.K.; Skulas-Ray, A.C.; Kris-Etherton, P.M. Recommended Intake of Fish and Fish Oils Worldwide. In Fish and Fish Oil in Health and Disease Prevention; Elsevier: Amsterdam, Netherlands, 2016; pp. $27-48$.

44. Sygnowska, E.; Waśkiewicz, A.; Głuszek, J.; Kwaśniewska, M.; Biela, U.; Kozakiewicz, K.; Zdrojewski, T.; Rywik, S. Kardiologia Polska; Polskie Towarzystwo Kardiologiczne: Warszawa, Polska, 2005.

45. Niedźwiedzka, E.; Wądołowska, L. Analiza urozmaicenia spożycia żywności w kontekście statusu socjoekonomicznego polskich osób starszych Analysis of food intake variety in relation to the socio-economic status of elderly Polish citizens. Probl. Hig. Epidemiol. 2010, 91, 576-584.

46. Mozaffarian, D.; Pischon, T.; Hankinson, S.E.; Rifai, N.; Joshipura, K.; Willett, W.C.; Rimm, E.B. Dietary intake of trans fatty acids and systemic inflammation in women. Am. J. Clin. Nutr. 2004, 79, 606-612. [CrossRef] [PubMed]

47. Dawczynski, C.; Massey, K.A.; Ness, C.; Kiehntopf, M.; Stepanow, S.; Platzer, M.; Grün, M.; Nicolaou, A.; Jahreis, G. Randomized placebo-controlled intervention with n-3 LC-PUFA-supplemented yoghurt: Effects on circulating eicosanoids and cardiovascular risk factors. Clin. Nutr. 2013, 32, 686-696. [CrossRef] [PubMed]

48. Harris, W.S.; Luo, J.; Pottala, J.V.; Margolis, K.L.; Espeland, M.A.; Robinson, J.G. Red Blood Cell Fatty Acids and Incident Diabetes Mellitus in the Women's Health Initiative Memory Study. PLoS ONE 2016, 11, e0147894. [CrossRef] [PubMed] 
49. Flock, M.R.; Skulas-Ray, A.C.; Harris, W.S.; Etherton, T.D.; Fleming, J.A.; Kris-Etherton, P.M. Determinants of erythrocyte omega-3 fatty acid content in response to fish oil supplementation: A dose-response randomized controlled trial. J. Am. Heart Assoc. 2013, 2, e000513. [CrossRef] [PubMed]

50. Harris, W.S.; Pottala, J.V.; Varvel, S.A.; Borowski, J.J.; Ward, J.N.; McConnell, J.P. Erythrocyte omega-3 fatty acids increase and linoleic acid decreases with age: Observations from 160,000 patients. Prostaglandins Leukot. Essent. Fat. Acids 2013, 88, 257-263. [CrossRef] [PubMed]

51. Kroger, J.; Zietemann, V.; Enzenbach, C.; Weikert, C.; Jansen, E.H.; Doring, F.; Joost, H.-G.; Boeing, H.; Schulze, M.B. Erythrocyte membrane phospholipid fatty acids, desaturase activity, and dietary fatty acids in relation to risk of type 2 diabetes in the European Prospective Investigation into Cancer and Nutrition (EPIC)-Potsdam Study. Am. J. Clin. Nutr. 2011, 93, 127-142. [CrossRef] [PubMed]

52. Vessby, B.; Gustafsson, I.-B.; Tengblad, S.; Berglund, L. Indices of fatty acid desaturase activity in healthy human subjects: Effects of different types of dietary fat. Br. J. Nutr. 2013, 110, 871-879. [CrossRef] [PubMed]

53. Wood, K.E.; Lau, A.; Mantzioris, E.; Gibson, R.A.; Ramsden, C.E.; Muhlhausler, B.S. A low omega-6 polyunsaturated fatty acid (n-6 PUFA) diet increases omega-3 (n-3) long chain PUFA status in plasma phospholipids in humans. Prostaglandins Leukot. Essent. Fat. Acids 2014, 90, 133-138. [CrossRef] [PubMed]

54. Wood, K.E.; Mantzioris, E.; Gibson, R.A.; Ramsden, C.E.; Muhlhausler, B.S. The effect of modifying dietary LA and ALA intakes on omega-3 long chain polyunsaturated fatty acid (n-3 LCPUFA) status in human adults: A systematic review and commentary. Prostaglandins Leukot. Essent. Fat. Acids 2015, 95, 47-55. [CrossRef] [PubMed]

55. Matthan, N.R.; Ooi, E.M.; Van Horn, L.; Neuhouser, M.L.; Woodman, R.; Lichtenstein, A.H. Plasma phospholipid fatty acid biomarkers of dietary fat quality and endogenous metabolism predict coronary heart disease risk: A nested case-control study within the Women's Health Initiative observational study. J. Am. Heart Assoc. 2014, 3. [CrossRef] [PubMed]

56. Mayneris-Perxachs, J.; Guerendiain, M.; Castellote, A.I.; Estruch, R.; Covas, M.I.; Fitó, M.; Salas-Salvadó, J.; Martínez-González, M.A.; Aros, F.; Lamuela-Raventós, R.M.; et al. Plasma fatty acid composition, estimated desaturase activities, and their relation with the metabolic syndrome in a population at high risk of cardiovascular disease. Clin. Nutr. 2014, 33, 90-97. [CrossRef] [PubMed]

57. Warensjö, E.; Öhrvall, M.; Vessby, B. Fatty acid composition and estimated desaturase activities are associated with obesity and lifestyle variables in men and women. Nutr. Metab. Cardiovasc. Dis. 2006, 16, 128-136. [CrossRef] [PubMed]

58. Warensjö, E.; Sundström, J.; Lind, L.; Vessby, B. Factor analysis of fatty acids in serum lipids as a measure of dietary fat quality in relation to the metabolic syndrome in men1-3. Am. J. Clin. Nutr. 2006, 84, 442-448. [CrossRef] [PubMed]

59. Kabagambe, E.K.; Tsai, M.Y.; Hopkins, P.N.; Ordovas, J.M.; Peacock, J.M.; Borecki, I.B.; Arnett, D.K. Erythrocyte fatty acid composition and the metabolic syndrome: A National Heart, Lung, and Blood Institute GOLDN study. Clin. Chem. 2008, 54, 154-162. [CrossRef] [PubMed]

60. Huang, T.; Bhulaidok, S.; Cai, Z.; Xu, T.; Xu, F.; Wahlqvist, M.L.; Li, D. Plasma phospholipids n-3 polyunsaturated fatty acid is associated with metabolic syndrome. Mol. Nutr. Food Res. 2010, 54, 1628-1635. [CrossRef] [PubMed]

61. Cinti, D.L.; Cook, L.; Nagi, M.N.; Suneja, S.K. The fatty acid chain elongation system of mammalian endoplasmic reticulum. Prog. Lipid Res. 1992, 31, 1-51. [CrossRef]

62. Obukowicz, M.G.; Welsch, D.J.; Salsgiver, W.J.; Martin-Berger, C.L.; Chinn, K.S.; Duffin, K.L.; Raz, A.; Needleman, P. Novel, selective delta6 or delta5 fatty acid desaturase inhibitors as antiinflammatory agents in mice. J. Pharmacol. Exp. Ther. 1998, 287, 157-166. [PubMed]

63. Lemaitre, R.N.; Tanaka, T.; Tang, W.; Manichaikul, A.; Foy, M.; Kabagambe, E.K.; Nettleton, J.A.; King, I.B.; Weng, L.-C.; Bhattacharya, S.; et al. Genetic loci associated with plasma phospholipid n-3 fatty acids: A meta-analysis of genome-wide association studies from the CHARGE Consortium. PLoS Genet. 2011, 7, e1002193. [CrossRef] [PubMed]

64. Domei, T.; Yokoi, H.; Kuramitsu, S.; Soga, Y.; Arita, T.; Ando, K.; Shirai, S.; Kondo, K.; Sakai, K.; Goya, M.; et al. Ratio of serum n-3 to n-6 polyunsaturated fatty acids and the incidence of major adverse cardiac events in patients undergoing percutaneous coronary intervention. Circ. J. 2012, 76, 423-429. [CrossRef] [PubMed] 
65. Warensjö, E.; Sundström, J.; Vessby, B.; Cederholm, T.; Risérus, U. Markers of dietary fat quality and fatty acid desaturation as predictors of total and cardiovascular mortality: A population-based prospective study. Am. J. Clin. Nutr. 2008, 88, 203-209. [CrossRef] [PubMed]

66. Das, U.N. Essential fatty acids: Biochemistry, physiology and pathology. Biotechnol. J. 2006, 1, 420-439. [CrossRef] [PubMed]

67. Do, H.J.; Chung, H.K.; Moon, J.; Shin, M.-J. Relationship between the estimates of desaturase activities and cardiometabolic phenotypes in Koreans. J. Clin. Biochem. Nutr. 2011, 49, 131-135. [CrossRef] [PubMed]

68. Vessby, B.; Tengblad, S.; Lithell, H. Insulin sensitivity is related to the fatty acid composition of serum lipids and skeletal muscle phospholipids in 70-year-old men. Diabetologia 1994, 37, 1044-1050. [CrossRef] [PubMed]

69. Liu, Y.; Medeiros, L.; Revesz, E.; O’Dorisio, T. Desaturation Function Does Not Decline after Menopause in Human Females. Horm. Metab. Res. 2000, 32, 26-32. [CrossRef] [PubMed]

70. Medeiros, L.C.; Liu, Y.W.; Park, S.; Chang, P.H.; Smith, A.M. Insulin, but not estrogen, correlated with indexes of desaturase function in obese women. Horm. Metab. Res. 1995, 27, 235-238. [CrossRef] [PubMed]

71. James, M.J.; Ursin, V.M.; Cleland, L.G. Metabolism of stearidonic acid in human subjects: Comparison with the metabolism of other $\mathrm{n}-3$ fatty acids. Am. J. Clin. Nutr. 2003, 77, 1140-1145. [CrossRef] [PubMed]

72. Sands, S.A.; Reid, K.J.; Windsor, S.L.; Harris, W.S. The impact of age, body mass index, and fish intake on the EPA and DHA content of human erythrocytes. Lipids 2005, 40, 343-347. [CrossRef] [PubMed]

73. Cao, J.; Schwichtenberg, K.A.; Hanson, N.Q.; Tsai, M.Y. Incorporation and Clearance of Omega-3 Fatty Acids in Erythrocyte Membranes and Plasma Phospholipids. Clin. Chem. 2006, 52, 2265-2272. [CrossRef] [PubMed]

74. Jump, D.B. Dietary polyunsaturated fatty acids and regulation of gene transcription. Curr. Opin. Lipidol. 2002, 13, 155-164. [CrossRef] [PubMed]

(C) 2018 by the authors. Licensee MDPI, Basel, Switzerland. This article is an open access article distributed under the terms and conditions of the Creative Commons Attribution (CC BY) license (http://creativecommons.org/licenses/by/4.0/). 